\title{
6 \\ Structural and Chemical Modifications of Carbon Dioxide on Transport to the Deep Earth
}

\author{
Mario Santoro ${ }^{1,2}$, Federico A. Gorelli ${ }^{1,2}$, Kamil Dziubek ${ }^{2,3}$, Demetrio Scelta ${ }^{2,3}$, and Roberto Bini ${ }^{2,3,4}$
}

\begin{abstract}
The structural and chemical changes to which carbon dioxide is subjected with increasing pressure and temperature are discussed here with the purpose of following the modifications of this important geochemical material on proceeding from the Earth's surface down to the core-mantle boundary. The relevance of metastabilities, and then of kinetic controlled transformations, is evidenced in the P-T ranges characteristic of both molecular phases and extended covalently bonded structures. From a chemical point of view, this analysis highlights how the characterization of the melting of the extended structures would represent an important step to understand the role of this compound in the chemistry of the Earth's mantle.
\end{abstract}

\subsection{INTRODUCTION}

Carbon dioxide is central in our lives, as an atmospheric component, for its contribution to global warming (Jenkinson et al., 1991), and also for its pivotal role in the physics and chemistry of the Earth's interior. In fact, carbon entering the Earth's mantle through the subduction of carbon-bearing minerals at the ocean floors not only contributes to determining the physical properties of the mantle, it also affects the Earth's interior dynamics being involved in the processes that rule the heat transfer to the surface. The presence of $\mathrm{CO}_{2}$ has been indirectly revealed by inclusions within diamonds (Schrauder \& Navon, 1993) or mantle rock-forming minerals (Frezzotti $\&$ Touret, 2014), and it has been concluded that $\mathrm{CO}_{2}$-rich

${ }^{1}$ CNR-INO, Istituto Nazionale di Ottica, Firenze, Italy

${ }^{2}$ LENS, European Laboratory for Non-linear Spectroscopy, Firenze, Italy

${ }^{3}$ CNR-ICCOM, Istituto di Chimica dei Composti OrganoMetallici, Firenze, Italy

${ }^{4}$ Dipartimento di Chimica "Ugo Schiff", Università di Firenze, Firenze, Italy phases characterize the mantle (Dasgupta et al., 2011). Even though the distribution is rather inhomogeneous (Le Voyer et al., 2017) and the origin unclear, $\mathrm{CO}_{2}$ release from the most stable carbonates, such as magnesite at the core-mantle interface, could be one of the possible sources (Isshiki et al., 2004). This presence is relevant in many respects, such as favoring the silicate melting and the consequent cycling to the crust of different elements and volatiles (Dasgupta \& Hirschmann, 2006), or as an intermediate in the formation of diamonds (Maeda et al., 2017). In addition, the possibility that $\mathrm{CO}_{2}$ may react with silica under high pressure and temperature conditions, leading to the formation of new compounds, is also intriguing because of the different coordination of $\mathrm{C}$ and Si solid oxides under any given P-T conditions. A considerable computational (Aravindh et al., 2007; MoralesGarcía et al., 2013; Qu et al., 2016; Yong et al., 2018; Zhou et al., 2014) and experimental (Santoro et al., 2011; Santoro et al., 2014) effort has been made to solve this specific issue. Knowledge of the structural and chemical properties of $\mathrm{CO}_{2}$ with increasing pressure and temperature is therefore essential for understanding its role in the Earth's deep carbon cycle.

The phase diagram of $\mathrm{CO}_{2}$ is, among the simplest molecular systems, one of the most extensively studied 
(see Datchi \& Weck, 2014; Santoro \& Gorelli, 2006; Yoo, 2013; and references therein) but also one of the most debated, especially for pressures in excess of $60 \mathrm{GPa}$ where the formation of extended covalent solids is reported. Multiple controversies surround the nature and the stability region of the different $\mathrm{CO}_{2}$ crystalline polymorphs all lying below $45 \mathrm{GPa}$, the number and the differences among the covalent extended phases, the existence of intermediate bonding structures, and finally the stability of the high-pressure phases against dissociation. The reasons for disagreement among different authors can be attributed to two main factors. The first is the pronounced metastability of most of the $\mathrm{CO}_{2}$ phases, which implies the existence of large kinetic barriers to be overcome for accomplishing the structural transitions or the chemical transformations. In a simplified view, this process can be seen as a potential energy landscape where the pathway followed along a specific coordinate is characterized, from energetic point of view, by one or more local minima, each one related to an intermediate state. The height of the activation barriers separating these minima becomes the key parameter ruling the transformation towards thermodynamically stable species. The $\mathrm{P}-\mathrm{T}$ path followed in the experiment therefore becomes extremely important. The second issue is experimental in nature and is related to the challenging pressures and temperatures required for exploring the most controversial P-T regions. Experimental results also appear influenced by the sample environment, by hydrostatic or nonhydrostatic conditions, and by the way in which high temperatures are achieved.

In an attempt to provide a clear description of the present knowledge on these issues, we have organized this manuscript in the following manner. The first section will discuss the crystalline molecular phases; the second section will be concerned with the extended covalent structures. The knowledge of the melting line of both molecular and extended solid phases is rather limited, but the topic deserves a careful discussion, and it will be reviewed in the third section. The high-pressure hightemperature stability of carbon dioxide versus dissociation will be the last topic discussed.

\subsection{MOLECULAR CRYSTAL PHASES}

$\mathrm{CO}_{2}$ crystallizes at ambient temperature around 0.5 $\mathrm{GPa}$ into a primitive cubic cell $(\mathrm{Pa} \overline{3}, \mathrm{Z}=4)$ with the molecules aligned with the cell body diagonal. The evolution with pressure of the structural and vibrational properties of this phase, known as dry ice or phase I, have been recently extensively investigated (Giordano et al., 2010). Phase I transforms to the orthorhombic phase III ( $\mathrm{Cmca}$, $\mathrm{Z}=4$ ) with a sluggish transition starting just above 10 $\mathrm{GPa}$ and extending for several GPa (Aoki et al., 1994; Hanson, 1985; Iota \& Yoo, 2001). Almost no volume change exists between the two crystal structures that appear strictly related: in both phases the carbon atoms are located at the face-centered positions, differing only in the orientation of the molecules, which break the F-lattice symmetry (Aoki et al., 1994). A pairing of the molecules was speculated for this phase, leading to an anomalous high-strength molecular crystal (Yoo, 2013; Iota \& Yoo, 2001). Ab initio calculations challenged this conclusion; in fact, phase III resulted in a typical van der Waals crystal with a bulk modulus one order of magnitude smaller than that derived from the experimental data. This difference was ascribed to a disputable extrapolation to ambient pressure of the experimental data all acquired above $20 \mathrm{GPa}$ (Bonev et al., 2003).

A new phase (II) was observed by heating phase III above $16 \mathrm{GPa}$ and $500 \mathrm{~K}$ (Iota \& Yoo, 2001). This transition was not reversible on cooling and phase II was recovered at ambient temperature, thus suggesting the metastable nature of phase III. Raman and powder X-ray diffraction (XRD) data were interpreted also in this case as suggestive of a molecular pairing leading to a very large bulk modulus. These conclusions were demonstrated to be wrong by a synchrotron XRD study where the structure was solved using samples in quasihydrostatic conditions (Datchi et al., 2014). The structure was identified as tetragonal $\left(P 4_{2} / \mathrm{mnm} ; \mathrm{Z}=2\right)$ with molecular and intermolecular parameters typical of a molecular crystal in perfect agreement with density functional theory (DFT) predictions (Bonev et al., 2003). Interestingly, the computed thermodynamic domain of the $P 4_{2} / \mathrm{mnm}$ structure matched the entire P-T region of both phases II and III, thus supporting the suggested metastability of phase III.

Phase II transforms above $13 \mathrm{GPa}$ into a new phase, IV, once heated above $500 \mathrm{~K}$. The transformation temperature does not depend on pressure at least within the accuracy of the experimental data (Gorelli et al., 2004). This phase could be quenched to ambient temperature being stable upon compression and transforming to phase I below $10 \mathrm{GPa}$, thus suggesting that phase II is also metastable. Spectroscopic (Yoo et al., 2001) and XRD (Park et al., 2003) data were interpreted as suggestive of a bent molecule with elongated $\mathrm{C}=\mathrm{O}$ bonds, a structure intermediate between a molecular crystal and an extended covalent solid. Also in this case, these conclusions have been disputed by computational studies that found phase IV to be a typical molecular phase, with the $\mathrm{CO}_{2}$ molecule maintaining the bond length and the linearity of the isolated molecule (Bonev et al., 2003). IR and Raman data also support a typical molecular crystal structure (Gorelli et al., 2004). Recent structural determination based on synchrotron single crystal XRD data of phase IV indicated rhombohedral space group $R \overline{3} c$, with two linear inequivalent molecules in the unit cell and bond length close to that of the free molecule (Datchi et al., 2009). These findings were confirmed by first-principles calculations, 
which were also capable of perfectly reproducing the structure and the vibrational spectra, and by neutron diffraction data that confirmed the structure (Palaich et al., 2014; Palaich, 2016).

A new phase, indicated as phase VII, was discovered by compressing phase I above $640 \mathrm{~K}$ and $12 \mathrm{GPa}$ with a stability pressure range increasing with temperature (Giordano \& Datchi, 2007). The transition was found to be fully reversible. High-quality synchrotron XRD patterns acquired along the $726 \mathrm{~K}$ isotherm provided evidence of an orthorhombic unit cell $(\mathrm{Cmca}, \mathrm{Z}=4)$. Such structure was predicted to be stable at high pressure by different computational studies (Bonev et al., 2003; Kuchta \& Etters, 1988). The $\mathrm{C}=\mathrm{O}$ bond length determined for this phase is only slightly shorter than in phase I $(<2 \%)$, thus also supporting the molecular character of phase VII. Interestingly, the Cmca space group of phase VII is the same as that of the high-pressure phase III (Aoki et al., 1994; Yoo et al., 1999); symptomatic is also the fact that both phases form only from phase I. Indeed, whereas phase VII transforms upon compression to phase IV, it was never reobtained by decompressing phase IV. All these aspects led to the reasonable question if phase VII is thermodynamically stable. Confirmation of its thermodynamic stability was provided by the direct crystallization of this phase from the liquid at $827 \mathrm{~K}$ and 12.1 GPa (Giordano et al., 2007); however, when phase IV was produced by compressing phase VII, it could be isothermally decompressed until melting. In this way, the melting line of phase IV was also measured between 800 and $950 \mathrm{~K}$, becoming almost indistinguishable from that of phase VII. The energy difference between these phases is therefore small, and a large energy barrier prevents the IV-VII transformation.
Very recently, quasi-harmonic electronic structure calculations were able to accurately reproduce the structural and spectroscopic properties of the molecular crystal phases I, II, IV, and VII of carbon dioxide in a broad pressure range (Sontising et al., 2017). On the contrary, the structure proposed for phase III directly relaxes to phase VII and no other structure is found by crystal structure prediction. Phases III and VII are therefore suggested to be identical and kinetically accessible at ambient temperature and thermodynamically stable above $640 \mathrm{~K}$.

As a final comment, it should be remarked that the pressure evolution of the experimental volume data relative to phases I, II, III, IV, and VII is very similar and markedly different from that of phase V (see Figure 6.1), thus unequivocally pointing to the analogous (molecular) nature of all these phases and ruling out the misleading concept, both from a chemical and structural point of view, of the existence of intermediate structures between molecular and extended phases.

\subsection{EXTENDED COVALENT PHASES}

At pressures $>40 \mathrm{GPa}$ carbon dioxide transforms into silica-like extended covalent phases, thus removing, as occurs also for nitrogen (Eremets et al., 2004), the apparent ambient pressure anomalies with respect to the heavier elements of the group. The first evidence of an extended phase $(\mathrm{V})$ was the appearance in the Raman spectrum of a characteristic peak of the C-O-C stretching mode after laser heating phase III to $1800 \mathrm{~K}$ above 40 GPa (Iota et al., 1999). This new phase could be quenched at ambient temperature down to approximately $10 \mathrm{GPa}$ where it transformed to phase I (Datchi \& Weck, 2014).

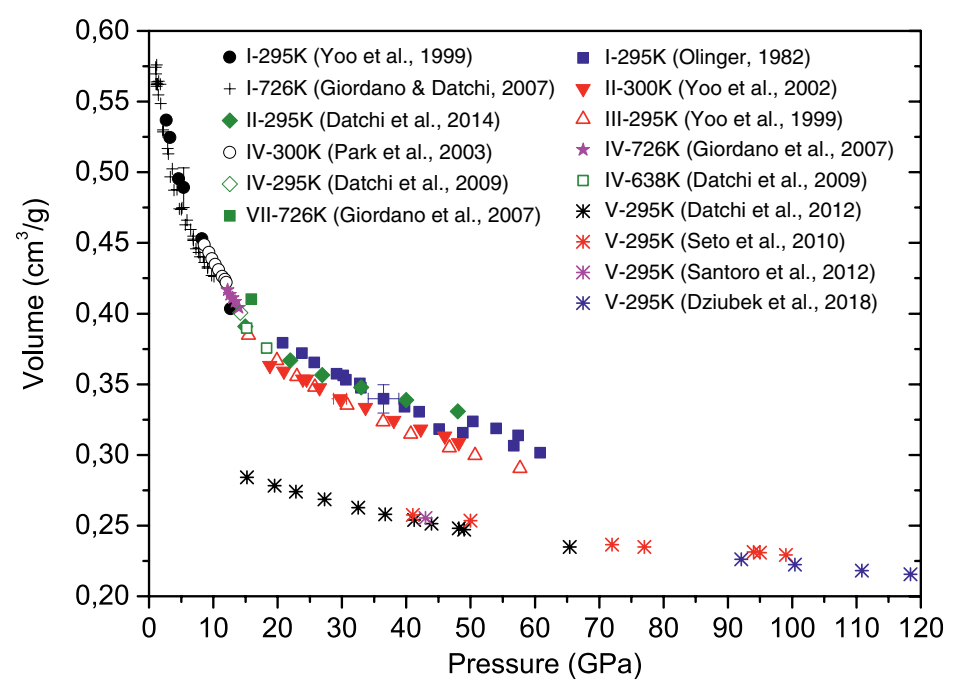

Figure 6.1 Experimental volume data of the extended phase $\mathrm{V}$ and of the stable and metastable molecular phases of crystalline $\mathrm{CO}_{2}$. See electronic version for color representation of the figures in this book. 
Lower pressure values have also been reported with phase $\mathrm{V}$ transforming directly to the fluid (Iota et al., 1999). Recently, phase $\mathrm{V}$ was claimed to be quenchable at ambient pressure and low temperature (Yong et al., 2016), but this conclusion was questioned because residual pressure was inferred by the frequency of the Raman modes of coexisting phase I (Datchi et al., 2017). The P-T conditions necessary for the attainment and its large stability range evidence the existence of a remarkably high energy barrier, which must be overcome to accomplish the chemical transformation from the molecular phases to phase V. This was recently demonstrated by synthesizing this phase just above $20 \mathrm{GPa}$ by laser heating phase IV using Ti as a catalyst (Sengupta et al., 2012). The temperature necessary for the formation of phase $\mathrm{V}$ found in two follow-up studies was considerably lower $(\mathrm{T}<1000$ K) (Santoro et al., 2004; Tschauner et al., 2001).

The crystal structure of phase $\mathrm{V}$ has been debated for more than one decade. The structure proposed on the basis of the first synchrotron XRD studies resembled those of the tridymite family with an orthorhombic $P 2_{1} 2_{1} 2_{1}$ unit cell and a bulk modulus of $365 \mathrm{GPa}$, therefore larger than stishovite (Yoo et al., 1999). These findings were not supported by a series of DFT computational works that predicted as stable phases $\alpha$-quartz (Serra et al., 1999) or a distorted $\beta$-cristobalite structure (Dong, Tomfohr, \& Sankey, 2000; Dong, Tomfohr, Sankey, Leinenweber, et al., 2000; Holm et al., 2000), with bulk modulus two to three times lower. The stability of the $\beta$-cristobalite structure was ascribed to the rigidity of the $\widehat{C O C}$ angles between connected tetrahedra $\left(120^{\circ}-125^{\circ}\right)$, to be compared with the wider analogous angles in silica $\left(145^{\circ}-150^{\circ}\right)$, thus speculating that the extended covalent phases of $\mathrm{CO}_{2}$ will not show the same abundant polymorphism as silica (Dong, Tomfohr, \& Sankey, 2000). The structure of phase $\mathrm{V}$ was first suggested (Seto et al., 2010) and then independently solved by two different groups (Datchi et al., 2012; Santoro et al., 2012). As predicted by DFT calculations, a partially collapsed $\beta$-cristobalite structure (space group $I \overline{4} 2 d$ ) was found, with carbon and oxygen atoms arranged in slightly distorted $\mathrm{CO}_{4}$ tetrahedra (tilted by $\sim 38^{\circ}$ about the $c$ axis) linked by oxygen atoms at the corners (Figure 6.2). Computed values of the bulk modulus were definitely smaller than the one reported for the tridymite structure ranging from 115 to $154 \mathrm{GPa}$ (Dong, Tomfohr, Sankey, Leinenweber, et al., 2000; Gohr et al., 2013; Holm et al., 2000; Oganov et al., 2008; Santoro et al., 2012). Experimentally, the first study provided a higher bulk modulus of $236 \mathrm{GPa}$ (Seto et al., 2010), but following XRD studies performed on decompression in both pure $\mathrm{CO}_{2}-\mathrm{V}$ samples and powder hydrostatically compressed with $\mathrm{He}$ allowed an accurate equation of state (EOS) from 8 to $65 \mathrm{GPa}$ providing a bulk modulus of $136 \mathrm{GPa}$ (Datchi et al., 2012). Higher pressure experimental volume data up to $100 \mathrm{GPa}$
(Seto et al., 2010) exceed this EOS by $\leq 5 \%$. Very recently, the synthesis of $\mathrm{CO}_{2}-\mathrm{V}$ above one megabar allowed the EOS extension up to $120 \mathrm{GPa}$ (Dziubek et al., 2018). The new volume data and those obtained with $\mathrm{CO}_{2}-\mathrm{V}$ compressed in He (Datchi et al., 2012) could be fitted with an EOS that further lowers the bulk modulus to a value (114 GPa) very close to that reported in Santoro et al. (2012). Interestingly, while the $a$ lattice parameter nicely fits the evolution of the lower pressure data, the $c$ parameter is considerably longer with the cla ratio increasing from 1.655 at $43 \mathrm{GPa}$ (Santoro et al., 2012) to 1.871 at $118 \mathrm{GPa}$ (Dziubek et al., 2018), thus getting closer to the fully collapsed structure, which expects a cla ratio equal to 2 (Figure 6.2).

The formation of an extended amorphous phase was evidenced by FTIR spectroscopy compressing phase III at ambient temperature (Santoro et al., 2006). This material, called a-carbonia in analogy with silica glass, forms when pressure exceeds $45 \mathrm{GPa}$ with a sluggish transformation that can be efficiently speeded up by moderate heating. The formation of an amorphous phase in the same pressure range was predicted by Serra et al. (1999). The XRD pattern and the vibrational spectra of a-carbonia are characterized by broad features that cannot be confused with those of phase V. First-principles molecular dynamics simulations have been capable to describe all the experimental signatures of this amorphous phase, providing insight into its bonding arrangement, which results in a mixture of three- and four-fold coordinated carbon atoms (Sun et al., 2009; Montoya et al., 2008). Interestingly, a-carbonia represents an excellent intermediate step for producing phase V. In fact, its strongest and characteristic IR absorption band is resonant with the $\mathrm{CO}_{2}$ laser lines so that it can be efficiently and cleanly heated without the need of absorbing materials (Santoro et al., 2012).

Other silica-like extended solids have been claimed but not confirmed by various authors. Although their Raman spectra are significantly different (Yoo et al., 2011), the common occurrence of metastability in the phase diagram of $\mathrm{CO}_{2}$, due to the high energy barriers characterizing most of the phase transitions, requires extreme caution in view of defining a reliable P-T path independent phase diagram. A stishovite-like phase VI (space group $\mathrm{P}_{2} / \mathrm{mnm}$ ), was claimed by the compression of molecular phase II above $50 \mathrm{GPa}$ and temperatures ranging between 530 and $650 \mathrm{~K}$ (Iota et al., 2007), with the carbon atoms having a six-fold coordination. Molecular dynamics simulations were not able to reproduce this phase, which was interpreted as due to an incomplete transformation (Sun et al., 2009). Recently, this P-T region was carefully investigated by synchrotron XRD with a resistively heated DAC in a P-T domain similar to that of Iota et al. (2007), without finding evidence of its existence (Santoro, M. unpublished data). Two other phases that appear as 

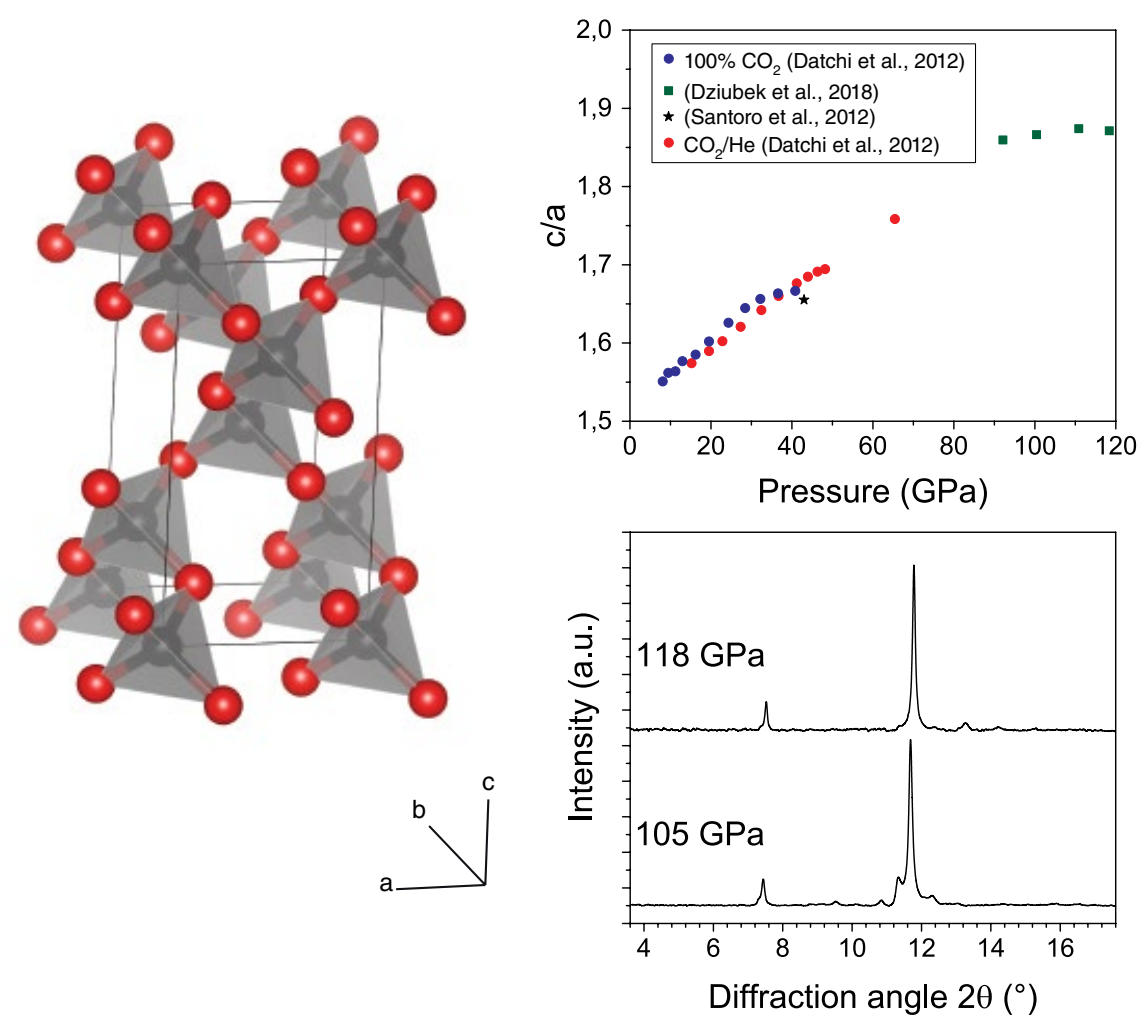

Figure 6.2 Left: refined structure of $\mathrm{CO}_{2}-\mathrm{V}$ at $118 \mathrm{GPa}$. Right: in the lower panel the XRD patterns measured (wavelength of $0.3738 \AA$ ) after two consecutive laser heating cycles $(2700 \mathrm{~K})$ are reported. The annealing effects leading to the disappearance of spurious peaks are evident in the second (118 GPa) pattern. In the top panel the c/a ratio of phase $V$ measured in different experiments is reported. See electronic version for color representation of the figures in this book.

kinetically frozen intermediate structures are coesite-like carbon dioxide $\left(\mathrm{c}-\mathrm{CO}_{2}\right)$, obtained only by laser-heating quenched phase VI (Sengupta \& Yoo, 2010), and $\mathrm{CO}_{2}-$ VIII, claimed to coexist with phase $\mathrm{V}$ above $50 \mathrm{GPa}$ and at undefined temperature (lower than that necessary for the synthesis of phase V) (Sengupta \& Yoo, 2009).

Upon laser heating to $1700-1800 \mathrm{~K}$ above $85 \mathrm{GPa}$, the extended phases $\mathrm{CO}_{2}-\mathrm{V}, a$-carbonia, and the alleged phase VI are reported to transform into a new extended ionic solid (i-CO $\mathrm{CO}_{2}$ ) that is stable during decompression down to $10 \mathrm{GPa}$, where phase I is obtained (Yoo et al., 2011). The Raman spectrum of this material is claimed to match that of orthorhombic $(P 2,2,2)$ carbonyl carbonate $\left(\mathrm{COCO}_{3}\right)$. These results have been recently challenged by the synthesis of $\mathrm{CO}_{2}-\mathrm{V}$ above $1 \mathrm{Mbar}$ (Dziubek et al., 2018). In this work, phase $\mathrm{V}$ was synthesized by laser heating amorphized $\mathrm{CO}_{2}$ at $85 \mathrm{GPa}$ and $2700 \mathrm{~K}$; the quenched phase $\mathrm{V}$ was laser heated again at the same temperature and a pressure just above $1 \mathrm{Mbar}$, the same conditions that should ensure the formation of $\mathrm{i}-\mathrm{CO}_{2}$, but the only effect was the obtainment of a high-quality perfectly annealed phase V (Figure 6.2). According to these results, phase $\mathrm{V}$ seems therefore to be the only thermodynamic stable extended phase between 120 and $10 \mathrm{GPa}$, whereas all the other extended phases are likely incompletely transformed materials, i.e local minima in the energy landscape.

\subsection{MELTING LINE}

The presence of fluid $\mathrm{CO}_{2}$ has been reported to be potentially important in establishing the chemicophysical properties of the mantle as well as the depth of its partial melting (Dasgupta \& Hirschmann, 2006; Isshiki et al., 2004). Therefore, one of the central issues regarding the presence and the chemistry of $\mathrm{CO}_{2}$ in the depth is the knowledge of the melting line of both molecular and extended phases.

The melting line was determined by visual observation, fluid-solid (phase I) coexistence in the 300-800 K temperature range, and pressures up to $11 \mathrm{GPa}$ (Giordano et al., 2006). In almost the same P-T range, the structure of liquid $\mathrm{CO}_{2}$ was recently investigated by both synchrotron XRD and classical molecular dynamics simulations showing an anisotropic short-range structure (Datchi et al., 2016). This is essentially related to a change in the 
orientation of some molecules in the first shell from the characteristic quadrupole-driven $\mathrm{T}$-shape arrangement to a slipped parallel configuration. The amount of molecules assuming the latter configuration increases with pressure in close analogy to the structural arrangement evolution in the crystal phases, since the two relative orientations are those characterizing phase I (T-shape) and phases III and VII. The melting line has been further extended up to $950 \mathrm{~K}$ and $15 \mathrm{GPa}$, also looking for the fluid-solid coexistence (Giordano \& Datchi, 2007). The melting of phase I was observed up to $808 \mathrm{~K}(11.7 \mathrm{GPa})$, and further compression of phase I produced phase IV, whose decompression in the fluid allowed to determine the triple point between the liquid and phases I and IV at $800 \mathrm{~K}$ and $11.2 \mathrm{GPa}$. At $810 \mathrm{~K}(11.8 \mathrm{GPa})$, the crystal in equilibrium with the fluid was $\mathrm{CO}_{2}$-VII, whose melting line could be followed up to $920 \mathrm{~K}$. Compression of phase VII produced phase IV, which could be decompressed down to the melting so that the melting curve of phase IV could be extended up to $946 \mathrm{~K}$, becoming practically indistinguishable from that of phase VII. Extrapolation of the melting line and of the IV-VII phase boundary leads to a liquid-IV-VII triple point at about $1030 \mathrm{~K}$ and $18.4 \mathrm{GPa}$. A good agreement with these results was shown by a more recent laser heating study that extended the melting line up to $29.5 \mathrm{GPa}$ and $1600 \mathrm{~K}$ (Litasov et al., 2011). No additional extension of the melting line was possible because both phases II and III transformed to the extended phase $\mathrm{V}$ when heated above $35 \mathrm{GPa}$, and further heating of phase $V$ between 35 and $70 \mathrm{GPa}$ caused dissociation of carbon dioxide in $\epsilon$-oxygen and elemental carbon. A triple point between the fluid, phase IV, and the extended phase $\mathrm{V}$ was identified at $33 \mathrm{GPa}$ and 1720 $\mathrm{K}$. Another triple point between phase V, fluid, and dissociated $\mathrm{CO}_{2}$ is located around $35 \mathrm{GPa}$ and $1900 \mathrm{~K}$, thus making the stability region of extended $\mathrm{CO}_{2}$ extremely limited at the P-T conditions typical of the mantle. As already stated, this claim was recently confuted by producing $\mathrm{CO}_{2}-\mathrm{V}$ at pressures and temperatures largely exceeding these conditions (Dziubek et al., 2018).

Just as there is little available experimental data regarding the melting line at $\mathrm{P}-\mathrm{T}$ conditions relevant for the Earth's interior, so too are computational studies rather limited. $\mathrm{Ab}$ initio calculations determined a melting line exhibiting a monotonic evolution with a positive slope up to $140 \mathrm{GPa}$ (Boates et al., 2012). In addition, by evaluating the coordination number of carbon atoms with respect to oxygen, it was possible to identify the stability regions of molecular and associated (extended) liquids. A first-order molecular to extended liquid-liquid phase transition is located slightly below $50 \mathrm{GPa}$ at temperatures up to $3000 \mathrm{~K}$. The important consequence of this result is the possible presence of an extended solid in equilibrium with its fluid at the lowermost mantle conditions.
Interestingly, $\mathrm{CO}_{2}$ was found to be stable against dissociation up to $140 \mathrm{GPa}$ and temperatures of the order of 7000 $\mathrm{K}$. In a following report, the same group computed the melting curve up to $71 \mathrm{GPa}$ and the molecular to extended phase transition using a phase coexistence approach from free energy calculations (Teweldeberhan et al., 2013). The triple point between phase IV, extended phase V, and the liquid was computed at $31.8 \mathrm{GPa}$ and $1636 \mathrm{~K}$, in excellent agreement with the experimental data (Litasov et al., 2011). The melting line computed in this work lies at higher temperatures with respect to that computed in previous single-phase simulations (Boates et al., 2012). The intersection of the melting line with the Earth's geotherm occurs at about $40 \mathrm{GPa}$ and $2160 \mathrm{~K}$ but with a much-pronounced positive slope, so that the melting line is about $2000 \mathrm{~K}$ higher than the Earth's geotherm at $70 \mathrm{GPa}$. This is a relevant finding because it means that if deposits of pure carbon dioxide in the lower mantle exist, they occur in the form of extended phase V. Interestingly, the liquid in equilibrium with the extended phase $\mathrm{V}$ appears also as an extended liquid with a coordination continuously shifting from three to four in analogy with the amorphous extended solid phase (Montoya et al., 2008).

\subsection{CARBON DIOXIDE STABILITY VERSUS DISSOCIATION}

While it has been long established that, after water, carbon dioxide is the second most important volatile in the deep Earth, its role in carbon fluxes and mantle carbon content is far from being fully understood. As a matter of fact, all the analyses concerning storage of $\mathrm{CO}_{2}$ in deep reservoirs can be misleading if its chemical form is not taken into account. It is noteworthy that the existence of $\mathrm{CO}_{2}$-rich fluids, pivotal for major geodynamic processes such as carbon recycling, does not necessarily signify that deposits of pure carbon dioxide are found in the deep mantle. Carbon may be most likely fractionated between the free $\mathrm{CO}_{2}$ phases, $\mathrm{CO}_{2}$ dissolved as a stoichiometric component in silicate or carbonate melts, and minerals (carbonates, carbides or diamond). Indeed, decarbonation processes of subducted carbonates involving diamond formation from the mixtures of MORB and $\mathrm{CaCO}_{3}$, or $\mathrm{SiO}_{2}$ and $\mathrm{MgCO}_{3}$, at pressures above $30 \mathrm{GPa}$ and temperatures in excess of $3000 \mathrm{~K}$ were observed by Seto et al. (2008). Moreover, the reactivity of $\mathrm{SiO}_{2}+\mathrm{MgCO}_{3}$ mixtures was characterized in a large pressure range, evidencing the diamond formation above $80 \mathrm{GPa}$ and considerably lower temperatures $(\mathrm{T} \geq 1700$ K), as found by Maeda et al. (2017). Neither study provides direct evidence of $\mathrm{CO}_{2}$ formation and its successive dissociation. Actually, the two-step mechanism of the $\mathrm{CO}_{2}$ liberation from carbonates and the subsequent dissociation of $\mathrm{CO}_{2}$ was questioned as a plausible theory 
explaining the origin of ultra-deep diamonds (Kaminsky, 2017). What is more, recent theoretical studies indicate that direct decomposition of $\mathrm{CaCO}_{3}$ and $\mathrm{MgCO}_{3}$ is unfavorable over the lower mantle, putting into doubt the possibility of free $\mathrm{CO}_{2}$ existing at these depths (Pickard \& Needs, 2015; Santos et al., 2019). It should be also emphasized that possible chemical processes, involving either reactions of $\mathrm{CO}_{2}$ or carbonates with elemental iron of the Earth's outer core close to the core-mantle boundary (Dorfman et al., 2018; Martirosyan et al., 2019), or with hydrous iron minerals like goethite (Boulard et al., 2018), can dramatically change carbon speciation in the lower mantle. In light of these findings, drawing conclusions concerning the deep carbon cycle on the basis of high pressure-high temperature behavior of pure $\mathrm{CO}_{2}$ requires extreme care.

Having said that, the stability of $\mathrm{CO}_{2}$ with respect to dissociation was extensively studied as a major issue in the Earth's mantle chemistry. Dissociation into diamond and fluid oxygen would have important consequences on the oxidation state of the lower mantle because the $\mathrm{O}_{2}$ formation would consistently increase the oxygen fugacity with the consequent oxidation of surrounding rocks and metals (Litasov et al., 2011). Shock compression experiments report the dissociation of carbon dioxide above $4000 \mathrm{~K}$ in the 0-34 GPa pressure range (Nellis et al., 1991; Oehlschlaeger et al., 2005). On the other hand, the first experiments on pure carbon dioxide reporting dissociation in DAC were performed by Tschauner et al. (2001) using a $\mathrm{CO}_{2}$ laser as heating source without any absorber below $40 \mathrm{GPa}$, whereas at higher pressures ruby or quartz powders were necessary to reach temperatures between 2500 and $3000 \mathrm{~K}$. The formation of oxygen was evidenced in all the runs by the appearance of the characteristic signatures of the $\epsilon$ phase in the Raman spectra, with and without absorbers, when the temperature overcame 2500 $\mathrm{K}$. For temperatures not exceeding $1500 \mathrm{~K}$, the peaks of oxygen were absent and other peaks assigned to an unidentified phase of $\mathrm{CO}_{2}$ were observed. The instability boundary identified in this study is characterized by a negative slope and exhibits a linear evolution with pressure extending from $28 \mathrm{GPa}$ and $2800 \mathrm{~K}$ to $69 \mathrm{GPa}$ and $1700 \mathrm{~K}$. This boundary, although maintaining a very similar negative slope, was shifted to lower temperatures in Litasov et al. (2011). Here, the sample heating was realized through a near IR laser absorbed by iridium couplers. $\mathrm{CO}_{2}$ dissociated always from the extended phase $\mathrm{V}$ for pressures in excess of $35 \mathrm{GPa}$ and temperatures below $1900 \mathrm{~K}$. Both of these studies reporting the dissociation were limited to pressures $\leq 70 \mathrm{GPa}$.

These results contrast with the stability of phase $\mathrm{V}$ at $\mathrm{P}$ $\leq 40 \mathrm{GPa}$ and temperatures up to $3000 \mathrm{~K}$ (Park et al., 2003) and with later studies that reported the transformation at $85 \mathrm{GPa}$ and $1700 \mathrm{~K}$ from the extended phase $\mathrm{V}$ to the ionic compound identified as carbonyl carbonate (Yoo et al., 2011). In all these cases, the sample was indirectly laser heated using Pt, B, or ruby as absorbers. Both dissociation and the formation of an ionic compound are challenged by the most recent studies, where $\mathrm{CO}_{2}-\mathrm{V}$ was synthesized with a remarkable quality by laser heating carbonia between $85 \mathrm{GPa}$, the same pressure as in Yoo et al. (2011), and $115 \mathrm{GPa}$ at temperatures in the order of 2500 K (Dziubek et al., 2018). Remarkably, repeated heating cycles have the only effect of producing a better crystallinity of the extended phase $\mathrm{V}$, as expected in a normal thermal annealing process, and suggest that phase $\mathrm{V}$ is the thermodynamic stable phase in this portion of the phase diagram. Annealing effects could explain the difficulty to reproduce the synthesis of many of the claimed extended phases (VI, VIII, i- $\mathrm{CO}_{2}$ ) that are likely due to an incomplete transformation of the starting material.

As far as the dissociation is concerned, it is evident that in spite of the different P-T ranges covered, the extrapolation of the proposed instability boundaries (Litasov et al., 2011; Tschauner et al., 2001) to higher pressures suggests dissociation just above $1500 \mathrm{~K}$ for pressures in excess of $80 \mathrm{GPa}$. Two possible explanations are proposed to account for this discrepancy. The first one is related to the occurrence of a redox reaction where the carbon in $\mathrm{CO}_{2}$ is reduced to diamond or graphite while the metal, and specifically rhenium, is oxidized (Dziubek et al., 2018; Santamaria Perez, 2016a; Santamaria Perez, 2016b). Obviously, this does not explain the results obtained below $40 \mathrm{GPa}$ when $\mathrm{CO}_{2}$ was laser heated without any absorber (Tschauner et al., 2001). However, in this case the P-T conditions of the dissociation fall within the fluid stability range, where dissociation requires a substantial lower energy to occur. Another possible explanation is related to the different P-T range investigated. Dissociation could be prevented at higher pressures because it would require a molecularity increase, which is disfavoured with increasing pressure. It is also worth mentioning that one of the main arguments employed for supporting the dissociation is the lower molar volume of diamond plus $\epsilon-\mathrm{O}_{2}$ with respect to $\mathrm{CO}_{2}-\mathrm{V}$ above $22 \mathrm{GPa}$ (a difference of $\sim 6 \%$ around 100 GPa) (Litasov et al., 2011; Tschauner et al., 2001), thus indicating that in the absence of other transformation $\mathrm{CO}_{2}$ should decompose. However, these data were related to the EOS obtained by using the data from Yoo et al. (1999) based on the wrong tridymite structure, whereas the EOS related to the partially collapsed $\beta$-cristobalite structure, determined independently by different groups (Datchi et al., 2012; Dziubek et al., 2018), lies below the one of $\mathrm{C}+\epsilon-\mathrm{O}_{2}$ up to the Mbar range. DFT molecular dynamics simulations do not expect dissociation below temperatures of 6000-7000 K (Boates et al., 2012), and in 
fact at P-T conditions well above the proposed dissociation experimental boundary ( $70 \mathrm{GPa}$ and $3000 \mathrm{~K}$ ), phase $\mathrm{V}$ is thermodynamically preferred, with differences in the free energy largely exceeding uncertainties and approximations of the method (Teweldeberhan et al., 2013).

\subsection{CONCLUSIONS}

We have reviewed here the present knowledge about carbon dioxide under high pressure and high temperature up to the lowermost mantle conditions (Figure 6.3). The $\mathrm{P}-\mathrm{T}$ region where carbon dioxide exists as a molecular crystal $(\mathrm{P}<40 \mathrm{GPa}$ and $\mathrm{T}<1000 \mathrm{~K})$, although not as relevant for the deep Earth carbon cycle, is extremely important from a fundamental point of view. Among the five molecular phases that have been identified, the experimental data suggest that the only thermodynamic stable phases are likely I, IV, and VII. It should be remarked that in spite of being stable or metastable, all these phases are "normal" molecular crystals, in the sense that the molecular structure and the intermolecular interactions are substantially unaltered across the different phases. The observation of metastable phases over large P-T ranges evidences the presence of large energetic barriers and of several local minima in the ground state energy surface, remarking the importance of kinetic control over the transformation processes. Free-energy calculations of all the known experimental phases, particularly if temperature effects are taken into account, would greatly help to clarify the puzzling stability of $\mathrm{CO}_{2}$ molecular phases.

The kinetic control is even more important in the formation of the new silica-like extended phases. This chemical transformation occurs, depending on the temperature value, between 35 and $60 \mathrm{GPa}$. The stoichiometry of molecular and extended phases is the same so that the same energy surface is common to all these phases. Also for the extended covalent phases, an important dependence on the P-T path followed is evidenced, leading to a wealth of local minima and corresponding metastable structures. Among the proposed extended polymorphs, phase $\mathrm{V}$ appears as the only one thermodynamically stable extending from pressures characteristic of the molecular phases to at least $120 \mathrm{GPa}$. Phase V is characterized by a $\beta$-cristobalite structure composed by $\mathrm{CO}_{4}$ tetrahedra, linked by oxygen atoms at the corners, whose degree of distortion increases with pressure leading to a more compact crystal. The amorphous counterpart of phase V, carbonia, is composed by a mixture of three-fold and four-fold coordinated carbon atoms and forms at temperatures close to ambient and $\mathrm{P} \geq 45 \mathrm{GPa}$, representing a typical kinetically driven transformation with incomplete realization of the four-fold coordination of phase $\mathrm{V}$.

From the geochemical point of view, the main questions related to carbon dioxide concern the form in which $\mathrm{CO}_{2}$ exists along the geotherm and its chemical stability with respect to dissociation or to interaction with minerals.

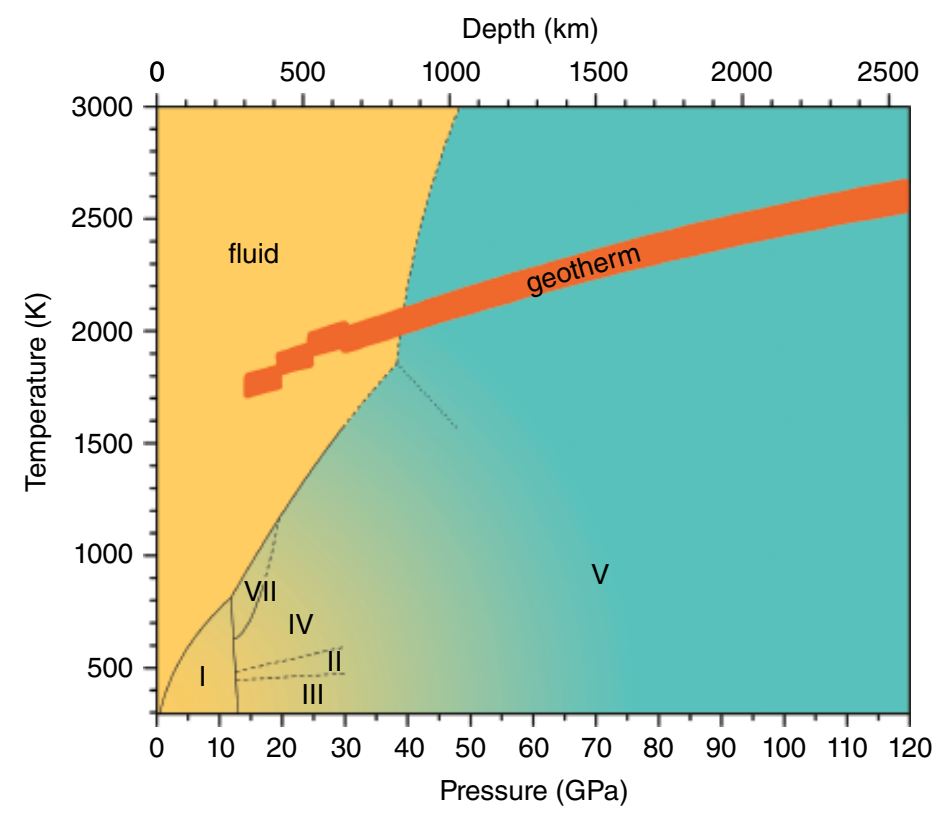

Figure 6.3 Overlap of the phase diagrams of molecular (yellow) and extended (cyan) $\mathrm{CO}_{2}$. The two colors have been chosen to mark the different chemical nature of the two systems. In both phase diagrams, only the thermodynamically stable phases (with the exception of phases II and III) have been reported. The blended area represents the P-T region where phase $\mathrm{V}$ can be quenched on decompression. The melting line is designed using experimental data up to 30 GPa (Giordano et al., 2006; Giordano \& Datchi, 2007; Litasov et al., 2011) and the computed data from Teweldeberhan (2013) at higher pressures. See electronic version for color representation of the figures in this book. 
Recent reports extended the stability range of phase $\mathrm{V}$ down to the lowermost mantle conditions $(\sim 2500 \mathrm{~km})$ where $\mathrm{CO}_{2}$ was supposed to dissociate into diamond and oxygen or possibly transform into carbonyl carbonate. The stability of this phase at these depths was already predicted by computational studies that expect the geotherm to fall within the range of stability of the extended phase $\mathrm{V}$ above $40 \mathrm{GPa}$. The presence of pure $\mathrm{CO}_{2}$ in the form of a covalently bonded extended solid down to the mantlecore boundary is extremely important from a chemical point of view. In fact, unless melting is induced by interaction with minerals, it is difficult to envisage an active role of $\mathrm{CO}_{2}$ in modifying the rheological properties and also the partial melting of mantle rocks at these depths. A very important issue not yet experimentally accessed is the evolution of the melting, or alternatively decomposition, line of phase $\mathrm{V}$. The melting is expected, according to computational studies, at remarkably higher temperatures than the geotherm for pressures in excess of $50 \mathrm{GPa}$; in addition, the nature of the melt could be crucial in determining the chemical activity of this species.

\section{ACKNOWLEDGMENTS}

This work was supported by the Deep Carbon Observatory.

\section{REFERENCES}

Aoki, K., Yamawaki, H., Sakashita, M., Gotoh, Y. \& Takemura, K. (1994). Crystal structure of the high-pressure phase of solid $\mathrm{CO}_{2}$. Science, 263, 356-358. https://doi.org/10.1126/ science. 263.5145.356

Aravindh, A. et al. (2007) $\mathrm{Si}_{\mathrm{x}} \mathrm{C}_{1-\mathrm{x}} \mathrm{O}_{2}$ alloys: A possible route to stabilize carbon-based silica-like solids? Solid State Commun., 144, 273276. https://doi.org/10.1016/j.ssc.2007.09.011

Boates, B., Teweldeberhan, A. M., \& Bonev, S. A. (2012). Stability of dense liquid carbon dioxide. Proc. Natl. Acad. Sci. USA, 109, 14808-14812. https://doi.org/10.1073/pnas. 1120243109

Bonev, S. A., Gygi, F., Ogitsu, T., \& Galli, G. (2003). High-pressure molecular phases of solid carbon dioxide. Phys. Rev. Lett., 91, 065501. https://doi.org/10.1103/PhysRevLett.91.065501

Boulard, E., Guyot, F., Menguy, N., Corgne, A., Auzende, A. L., Perrillat, J.-P., \& Fiquet, G. (2018). $\mathrm{CO}_{2}$-induced destabilization of pyrite-structured $\mathrm{FeO}_{2} \mathrm{Hx}$ in the lower mantle. National Science Review, 5, 870-877. https://doi.org/10.1093/ nsr/nwy032

Dasgupta, R., \& Hirschmann, M. M. (2006) Melting in the Earth's deep upper mantle caused by carbon dioxide. Nature, 440, 659-662, https://doi.org/10.1038/nature04612

Dasgupta, R., Mallik, A., Tsuno, K., Withers, A. C., Hirth, G., \& Hirschmann, M. M. (2011). Carbon-dioxide-rich silicate melt in the Earths upper mantle. Nature, 493, 211-215. https://doi.org/10.1038/nature11731

Datchi, F., Giordano, V. M., Munsch, P., \& Saitta, A. M. (2009). Structure of carbon dioxide phase IV: Breakdown of the intermediate bonding state scenario. Phys. Rev. Lett., 103, 185701. https://doi.org/10.1103/PhysRevLett.103.185701

Datchi, F., Mallick, B., Salamat, A., \& Ninet, S. (2012). Structure of polymeric carbon dioxide $\mathrm{CO}_{2}-\mathrm{V}$. Phys. Rev. Lett., 108, 125701. https://doi.org/10.1103/PhysRevLett.108.125701

Datchi, F., Mallick, B., Salamat, A., Rousse, G., Ninet, S., Garbarino, G., et al. (2014). Structure and compressibility of the high-pressure molecular phase II of carbon dioxide. Phys. Rev. B, 89, 144101.https://doi.org/10.1103/PhysRevB.89.144101

Datchi, F., Moog, M., Pietrucci, F., \& Saitta, A. M.(2017). Polymeric phase $\mathrm{V}$ of carbon dioxide has not been recovered at ambient pressure and has a unique structure. Proc. Natl. Acad. Sci. USA, 114, E656-E657. https://doi.org/10.1073/ pnas. 1619276114

Datchi, F., \& Weck, G. (2014). X-ray crystallography of simple molecular solids up to megabar pressures: Application to solid oxygen and carbon dioxide Z. Kristallogr., 229, 135157. DOI 10.1515/zkri-2013-1669

Datchi, F., Weck, G., Saitta, A. M., Raza, Z., Garbarino, G., Ninet, S. et al.(2016). Structure of liquid carbon dioxide at pressures up to $10 \mathrm{GPa}$. Phys. Rev. B: Condens. Matter Mater. Phys., 94, 014201. https://doi.org/10.1103/PhysRevB. 94.014201

Dong, J., Tomfohr, J. K., \& Sankey, O. F. (2000). Rigid intertetrahedron angular interaction of nonmolecular carbon dioxide solids. Phys. Rev. B: Condens. Matter Mater. Phys., 61, 5967-5971. https://doi.org/10.1103/PhysRevB.61.5967

Dong, J., Tomfohr, J. K., Sankey, O. F., Leinenweber, K., Somayazulu, M., \& McMillan, P. F. (2000). Investigation of hardness in tetrahedrally bonded nonmolecular $\mathrm{CO}_{2}$ solids by density-functional theory. Phys. Rev. B: Condens. Matter Mater. Phys., 62, 14685. https://doi.org/10.1103/PhysRevB.62.14685

Dorfman, S. M., Badro, J., Nabiei, F., Prakapenka, V. B, Cantoni, M., \& Gillet, P. (2018). Carbonate stability in the reduced lower mantle. Earth Planet. Sci. Lett., 489, 84-91. https://doi.org/10.1016/j.eps1.2018.02.035

Dziubek, K. F., Ende, M., Scelta, D., Bini, R., Mezouar, M., Garbarino, G., \& Miletich, R. (2018). Crystalline polymeric carbon dioxide stable at megabar pressures. Nat. Comm., 9, 3148. https://doi.org/10.1038/s41467-018-05593-8

Eremets, M. I., Gavriliuk, A. G., Trojan, J. A., Dzivenko, D. A., \& Boeler, R. (2004). Single-bonded cubic form of nitrogen. Nat. Mater., 3, 558-563. https://doi.org/10.1038/nmat1146

Frezzotti, M. L., \& Touret, J. L. R. (2014). $\mathrm{CO}_{2}$, carbonate-rich melts, and brines in the mantle. Geoscience Frontiers, 5, 697710. https://doi.org/10.1016/j.gsf.2014.03.014

Giordano, V. M., \& Datchi, F. (2007). Molecular carbon dioxide at high pressure and high temperature. EPL, 77, 46002. https://doi.org/10.1209/0295-5075/77/46002

Giordano, V. M., Datchi, F., \& Dewaele, A. (2006). Melting curve and fluid equation of state of carbon dioxide at high pressure and high temperature. J. Chem. Phys., 125, 054504. https://doi.org/10.1063/1.2215609

Giordano, V. M., Datchi, F., Gorelli, F. A., \& Bini, R. (2010). Equation of state and anharmonicity of carbon dioxide phase I up to $12 \mathrm{GPa}$ and $800 \mathrm{~K}$. J. Chem. Phys., 133, 144501. https://doi.org/10.1063/1.3495951

Gohr, S., Grimme, S., Shnel, T., Paulus, B., \& Schwerdtfeger, P. (2013). Pressure dependent stability and structure of carbon dioxide: A density functional study including long-range 
corrections. J. Chem. Phys., 139, 174501. https://doi.org/ $10.1063 / 1.4826929$

Gorelli, F. A., Giordano, V. M., Salvi, P. R., \& Bini, R. (2004). Linear carbon dioxide in the high-pressure high-temperature crystalline phase IV. Phys. Rev. Lett., 93, 205503. https://doi. org/10.1103/PhysRevLett.93.205503

Hanson, R. C. (1985). A new high-pressure phase of solid carbon dioxide. J. Phys. Chem., 89 (21), 4499-4501. https:// doi.org/10.1021/j100267a019

Holm, B., Ahuja, R., Belonoshko, A., \& Johansson, B. (2000). Theoretical investigation of high pressure phases of carbon dioxide. Phys. Rev. Lett., 85, 1258-1261. https://doi. org/10.1103/PhysRevLett.85.1258

Iota, V., \& Yoo, C. S. (2001). Phase diagram of carbon dioxide: Evidence for a new associated phase. Phys. Rev. Lett., 86, 5922-5925. https://doi.org/10.1103/PhysRevLett.86.5922

Iota, V., Yoo, C. S., \& Cynn, H. (1999). Quartzlike carbon dioxide: An optically nonlinear extended solid at high pressures and temperatures. Science, 283, 1510-1513. https://doi. org/10.1126/science.283.5407.1510

Iota, V., Yoo, C.-S., Klepeis, J.-H., Jenei, Z., Evans, W., \& Cynn, H. (2007). Six-fold coordinated carbon dioxide VI. Nat. Mater., 6, 34-38. https://doi.org/10.1038/nmat 1800

Isshiki, M., Irifune, T., Hirose, K., Ono, S., Ohishi, Y., Watanuki, T., et al. (2004). Stability of magnesite and its high-pressure form in the lowermost mantle. Nature, 427, 60-62. https:// doi.org/10.1038/nature02181

Jenkinson, D. S., Adams, D. E., \& Wild, A. (1991). Model estimates of $\mathrm{CO}_{2}$ emissions from soil in response to global warming. Nature, 351, 304-306. https://doi.org/10.1038/ $351304 \mathrm{a} 0$

Kaminsky, F. V. (2017). The Earth's Lower Mantle: Composition and Structure. Springer Geology, doi 10.17/978-3-319-55684-0.

Kuchta, B. \& Etters, R. D. (1988). Prediction of a high-pressure phase transition and other properties of solid $\mathrm{CO}_{2}$ at low temperatures. Phys. Rev. B, 38, 6265-6269. https://doi. org/10.1103/PhysRevB.38.6265

Le Voyer, M., Kelley, K. A., Cottrell, E., \& Haur, E. H (2017). Heterogeneity in mantle carbon content from $\mathrm{CO}_{2}$-undersaturated basalts. Nat. Comm., 8, 14062. https://doi.org/10.1038/ ncomms 14062

Litasov, K. D., Goncharov, A. F., \& Hemley, R. J. (2011). Crossover from melting to dissociation of $\mathrm{CO}_{2}$ under pressure: Implications for the lower mantle. Earth and Planetary Science Letters, 309, 318-323. https://doi. org/10.1016/j.epsl.2011.07.006

Maeda, F. A., Ohtani, E., Kamada, S., Sakamaki, T., Hirao, N., \& Ohishi, Y. (2017). Diamond formation in the deep lower mantle: a high-pressure reaction of $\mathrm{MgCO}_{3}$ and $\mathrm{SiO}_{2}$. Sci. Rep., 7, 40602. https://doi.org/10.1038/srep40602

Martirosyan, N. S., Litasov, K. D., Lobanov, S. S., Goncharov, A. F., Shatskiy, A., Ohfuji, H., \& Prakapenka, V. (2019). The $\mathrm{Mg}$-carbonate-Fe interaction: Implication for the fate of subducted carbonates and formation of diamond in the lower mantle. Geoscience Frontiers, 10, 1449-1458. https://doi. org/10.1016/j.gsf.2018.10.003

Montoya, J. A., Rousseau, R., Santoro, M., Gorelli. F. A., \& Scandolo, S. (2008). Mixed threefold and fourfold carbon coordination in compressed $\mathrm{CO}_{2}$. Phys. Rev. Lett., 100, 163002. https://doi.org/10.1103/PhysRevLett.100.163002

Morales-García, A., Marqués, M., Menéndez, J. M., Santamaría-Pérez, D., Baonza, V. G., \& Recio J. M. (2013). First-principles study of structure and stability in SiCObased materials. Theor. Chem. Acc., 132, 1308. https://doi. org/10.1007/s00214-012-1308-6

Nellis, W. J., Mitchell, A. C., Ree, F. H., Ross, M., Holmes, N. C., Trainor, R. J., \& Erskine, D. J. (1991). Equation of state of shock-compressed liquids: Carbon dioxide and air. J. Chem. Phys., 95, 5268-5272. https://doi.org/10.1063/1.461665

Oehlschlaeger, M. A., Davidson, D. F., Jeffries, J. B., \& Hanson, R. K. (2005). Carbon dioxide thermal decomposition: Observation of incubation. Z. Phys. Chem., 219, 555-567. https://doi.org/10.1524/zpch.219.5.555.64325

Oganov, A. R., Ono, S., Ma, Y., Glass, C. W., \& Garcia, A. (2008). Novel high-pressure structures of $\mathrm{MgCO}_{3}, \mathrm{CaCO}_{3}$ and $\mathrm{CO}_{2}$ and their role in Earth's lower mantle. Earth Planet. Sci. Lett., 273, 38-47. https://doi.org/10.1016/j.eps1.2008. 06.005

Olinger, B. (1982). The compression of solid $\mathrm{CO}_{2}$ at $296 \mathrm{~K}$ to 10 GPa. J. Chem. Phys. ,77, 6255-6258. https://doi.org/10.1063/1. 443828

Palaich, S. Carbon in the Deep Earth: A Mineral Physics Perspective. PhD diss., University of California-Los Angeles, 2016.

Palaich, S., Tulk, C. A., Molaison, J. J.,Makhluf, A. R., Guthrie, M., Kavner, A., \& Manning, C. E. (2014) Structure and thermal behavior of $\mathrm{CO}_{2}-\mathrm{IV}$ at $18 \mathrm{GPa}$ from $300-625 \mathrm{~K}$. AGU Fall Meeting Abstracts. http://adsabs.harvard.edu/ abs/2014AGUFMMR33A4332P

Park, J.-H., Yoo, C. S., Iota, V., Cynn, H., Nicol, M. F., \& Le Bihan, T. (2003). Crystal structure of bent carbon dioxide phase IV. Phys. Rev. B: Condens. Matter Mater. Phys., 68, 014107. https://doi.org/10.1103/PhysRevB.68.014107

Pickard, C. J., \& Needs, R. J. (2015). Structures and stability of calcium and magnesium carbonates at mantle pressures. Phys. Rev. B,91,104101.https://doi.org/10.1103/PhysRevB.91.104101 Qu, B., Li, D., Wang, L., Wu, J., Zhou, R., Zhang, B., \& Zeng, X. C. (2016). Mechanistic study of pressure and temperature dependent structural changes in reactive formation of silicon carbonate. RSC Adv., 6, 26650. https://doi.org/10.1039/ c5ra21981g

Santamaria Perez, D., McGuire, C., Makhluf, A., Kavner, A., Chuliá Jordan, R., Forda, J. L. et al. (2016a). Strongly-driven $\mathrm{Re}+\mathrm{CO}_{2}$ redox reaction at high-pressure and high-temperature. Nat. Comm., 7, 13647. https://doi.org/10.1038/ncomms13647

Santamaria Perez, D., McGuire, C., Makhluf, A., Kavner, A., Chuliá Jordan, R., Forda, J. L. et al. (2016b). Exploring the chemical reactivity between carbon dioxide and three transition metal ( $\mathrm{Au}, \mathrm{Pt}$ and $\mathrm{Re}$ ) at high-pressure, high-temperature conditions. Inorg. Chem., 55, 10793-10799. https:// doi.org/10.1021/acs.inorgchem.6b01858

Santoro, M., \& Gorelli, F. A. (2006). High pressure solid state chemistry of carbon dioxide Chem. Soc. Rev., 35, 918-931. https://doi.org/10.1039/B604306M

Santoro, M., Gorelli, F. A., Bini, R., Haines, J., Cambon, O., Levelut, C. et al. (2012). Partially collapsed cristobalite 
structure in the non molecular phase $\mathrm{V}$ in $\mathrm{CO}_{2}$. Proc. Natl. Acad. Sci. USA, 109, 5176-5179. https://doi.org/10.1073/ pnas. 1118791109

Santoro, M., Gorelli, F. A., Bini, R., Ruocco, G., Scandolo, S., \& Crichton, W. A. (2006). Amorphous silica-like carbon dioxide. Nature, 441, 857-860. https://doi.org/10.1038/ nature 04879

Santoro, M., Gorelli, F. A., Bini, R., Salamat, A., Garbarino, G., Levelut, C., et al. (2014). Carbon enters silica forming a cristobalite-type $\mathrm{CO}_{2} \mathrm{SiO}_{2}$ solid solution. Nat. Comm., 5, 3761. https://doi.org/10.1038/ncomms4761

Santoro, M., Gorelli, F. A., Haines, J., Cambon, O., Levelut, C., \& Garbarino, G. (2011). Silicon carbonate phase formed from carbon dioxide and silica under pressure. Proc. Natl. Acad. Sci. USA, 108, 7689-7692. https://doi.org/10.1073/ pnas. 1019691108

Santoro, M., Lin, J. F., Mao, H. K., \& Hemley, R. J. (2004). In situ high P-T Raman spectroscopy and laser heating of carbon dioxide. J. Chem. Phys., 121, 2780-2787. https://doi. org/10.1063/1.1758936

Santos, S. S. M., Marcondes, M. L., Justo, J. F., \& Assali, L. V. C. (2019). Stability of calcium and magnesium carbonates at Earth's lower mantle thermodynamic conditions. Earth Planet. Sci. Lett., 506, 1-7. https://doi.org/10.1016/j.epsl.2018.10.030

Schrauder, M., \& Navon, O. (1993). Solid carbon dioxide in a natural diamond. Nature, 365, 42-44. https://doi. org/10.1038/365042a0

Sengupta, A., Kim, M., Yoo, C.-S., \& Tse, J. S. (2012). Polymerization of carbon dioxide: A chemistry view of molecular-to nonmolecular phase transitions. J. Phys. Chem. C, 116, 2061-2067. https://doi.org/10.1021/jp204373t

Sengupta, A., \& Yoo, C. S. (2009). Raman studies of molecularto-nonmolecular transitions in carbon dioxide at high pressures and temperatures. Phys. Rev. B: Condens. Matter Mater. Phys., 80, 014118. https://doi.org/10.1103/PhysRevB.80.014118

Sengupta, A., \& Yoo, C. S. (2010). Coesite-like $\mathrm{CO}_{2}$ : An analog to $\mathrm{SiO}_{2}$. Phys. Rev. B: Condens. Matter Mater. Phys., 82, 012105. https://doi.org/10.1103/PhysRevB.82.012105

Serra, S., Cavazzoni, C., Chiarotti, G. L., Scandolo, S., \& Tosatti, E. (1999). Pressure-induced solid carbonates from molecular $\mathrm{CO}_{2}$ by computer simulation. Science, 284, 788790. https://doi.org/10.1126/science.284.5415.788

Seto, Y., Hamane, D., Nagai, T., \& Fujino, K. (2008). Fate of carbonates within oceanic plates subducted to the lower mantle, and a possible mechanism of diamond formation. Phys. Chem. Miner., 35, 223-229. https://doi.org/10.1007/ s00269-008-0215-9

Seto, Y., Nishio-Hamane, D., Nagai, T., Sata, N., \& Fujino, K. (2010) Synchrotron X-ray diffraction study for crystal structure of solid carbon dioxide $\mathrm{CO}_{2}-\mathrm{V}$. J. Phys. Conf. Ser., 215, 012015. https://doi.org/10.1088/1742-6596/215/1/012015
Sontising, W., Heit, Y. N., McKinley, J. L., \& Beran, G. J. O. (2017). Theoretical predictions suggest carbon dioxide phases III and VII are identical. Chem. Sci., 8, 7374-7382. https:// doi.org/10.1039/C7SC03267F

Sun, J., Klug, D. D., Martonak, R., Montoya, J. A., Lee, M. S., Scandolo, S., \& Tosatti, E. (2009). High-pressure polymeric phases of carbon dioxide. Proc. Natl. Acad. Sci. USA, 106, 6077-6081. https://doi.org/10.1073/pnas. 0812624106

Teweldeberhan, A. M., Boates, B., \& Bonev, S. A. (2013). $\mathrm{CO}_{2}$ in the mantle: Melting and solid-solid phase boundaries. Earth and Planetary Science Letters, 373, 228-232. https:// doi.org/10.1016/j.eps1.2013.05.008

Tschauner, O. Mao, H. K., \& Hemley, R. J. (2001). New transformations of $\mathrm{CO}_{2}$ at high pressures and temperatures. Phys. Rev. Lett., 87, 075701. https://doi.org/10.1103/PhysRevLett. 87.075701

Yong, X., Liu, H., Wu, M., Yao, Y., Tse, J. S., Dias, R., \& Yoo, C.-S. (2016). Crystal structures and dynamical properties of dense $\mathrm{CO}_{2}$. Proc. Natl. Acad. Sci. USA, 113, 11110-11115. https://doi.org/10.1073/pnas.1601254113

Yong, X., Tse, J. S., \& Chen, J. (2018) Mechanism of chemical reactions between $\mathrm{SiO}_{2}$ and $\mathrm{CO}_{2}$ under mantle conditions. ACS Earth Space Chem., 2, 548555. https://doi.org/10.1021/ acsearthspacechem. $7 \mathrm{~b} 00144$

Yoo, C.-S. (2013). Physical and chemical transformations of highly compressed carbon dioxide at bond energies. Phys. Chem. Chem. Phys., 15, 7949-7966. https://doi.org/10.1039/ c3cp50761k

Yoo, C. S., Cynn, H., Gygi, F., Galli, G., Iota, V., Nicol, M. F. et al. (1999). Crystal structure of carbon dioxide at high pressure: Superhard polymeric carbon dioxide. Phys. Rev. Lett., 83, 5527-5530. https://doi.org/10.1103/PhysRevLett. 83.5527

Yoo, C. S., Iota, V., \& Cynn, H. (2001). C.S. Yoo, V. Iota, and H. Cynn nonlinear carbon dioxide at high pressures and temperatures. Phys. Rev. Lett., 86, 444 447. https://doi.org/10.1103/ PhysRevLett.86.444

Yoo, C. S., Kohlmann, H., Cynn, H., Nicol, M. F., Iota, V., \& LeBihan, T. (2002). Crystal structure of pseudo-six-fold carbon dioxide phase II at high pressures and temperatures. Phys. Rev. B: Condens. Matter Mater. Phys., 65, 104103. https://doi.org/10.1103/PhysRevB.65.104103

Yoo, C.-S., Sengupta, A., \& Kim, M. (2011). Carbon dioxide carbonates in the Earth's mantle: Implications to the deep carbon cycle. Angew. Chem. Int. Ed., 50, 11219-11222. https:// doi.org/10.1002/anie.201104689

Zhou, R., Qu, B., Dai, J., \& Zeng, X. C. (2014) Unraveling crystalline structure of high-pressure phase of silicon carbonate. Phys. Rev. X, 4, 011030. https://doi.org/10.1103/ PhysRevX.4.011030 